\title{
Identification of microbial methane sources and sinks in an aquifer-fed continental wetland with a multi- omics approach
}

\author{
SARAH COFFINET ${ }^{1}$, ALEXIS DUFRESNE ${ }^{1}$, ACHIM \\ QUAISER $^{1}$, LAURENT LONGUEVERGNE ${ }^{1}$, KAI-UWE \\ HINRICHS $^{2}$ AND ANNIET LAVERMAN ${ }^{1}$ \\ ${ }^{1}$ University of Rennes 1 / CNRS \\ ${ }^{2}$ University of Bremen \\ Presenting Author: sarah.coffinet@univ-rennes1.fr
}

Vegetated continental wetlands are responsible for $20 \%$ of global methane emissions. Wetlands encompass very diverse ecosystems, from ponds to peatlands. In mid-latitudes, temporary flooded lowlands are very common, due to the seasonal discharge of water from rivers or groundwater but their contribution to the global methane budget is unknown. These sites experience temporal successions of oxic and anoxic conditions and the impact of these dynamic redox conditions on the microbial communities is poorly understood. In particular, methanogenesis typically requires anoxic conditions while aerobic methanotrophy can oxidize large amounts of methane before it reaches the atmosphere. This study is set on the Ploemeur-Guidel hydrogeological observatory, where a wetland is created by the seasonal discharge of deep anoxic and iron-rich groundwater. Batch incubations revealed high potential rates of methane production at depth, reaching up to $3.4 \mu \mathrm{mol} \mathrm{cm}{ }^{-3} \mathrm{~d}^{-1}$. To shed light on the impact of transient redox conditions on methane cycling by microbial communities, a metagenomics and metatranscriptomics analysis was conducted in combination with the analysis of the carbon isotopic composition of microbial intact polar lipids. Four one-meter-long cores were recovered along a transect from the inner to the outer side of the wetland and samples were collected every 20 to $30 \mathrm{~cm}$ along each core. Preliminary results of the omics study showed clear taxon stratification with depth. The proportion of reads classified to archaeal taxa increased with depth and reached up to $15 \%$ of the total analyzed reads at one meter below the surface. Archaeal communities appeared to be dominated by Candidatus Bathyarchaeota since up to $60 \%$ of the archaeal reads could be classified to this phylum. The proportion of reads affiliated with methanotrophic communities was highest near the surface with about $1.5 \%$ of the total reads decreasing to $\sim 0.5 \%$ at one meter depth. Investigation of genes related to methane cycling combined with carbon isotopic analysis of microbial lipids will be performed to identify the major metabolic pathways responsible for methane production and consumption in the ecosystem. 